\title{
Stigmatising views towards individuals with eating disorders: trends and associations from 1998 to 2008 using a repeated cross-sectional design
}

Jennifer Guy, Helen Bould, Glyn Lewis and Francesca Solmi

\section{Background}

Eating disorders are stigmatised. Little is known about whether stigma has decreased over time and which groups hold more stigmatising beliefs.

\section{Aims}

To explore whether stigma towards eating disorders has changed between 1998 and 2008 and whether it varies by sociodemographic characteristics.

\section{Method}

We used the Office for National Statistics Omnibus surveys 1998 and 2008. As outcomes, we selected four questions eliciting participants' views on issues of blame and ability to recover, and compared their mean scores across eating disorders, depression and alcohol dependence in both years. We used multivariable linear regressions to investigate associations between sociodemographic characteristics and each stigma domain.

\section{Results}

In total, 2720 participants had data on all variables of interest. Compared with 1998, in 2008 stigmatising views towards eating disorders improved. In both years, participants believed it was easier to recover from eating disorders than depression or alcohol dependence. Respondents believed people with eating disorders were more to blame for their condition than those with depression, but less than those with alcohol dependence. Men, those with less formal education, and those from ethnic minority backgrounds were more likely to place greater blame on individuals for their mental illness. Men were more likely than women to think it was possible to recover from an eating disorder.

\section{Conclusions}

Stigmatising attitudes towards people with eating disorders have improved over time, but are still greater than those observed for other mental illnesses. Improving eating disorder mental health literacy could help to reduce these negative views and lead to improved quality of life, greater help-seeking and better prognosis.

\section{Keywords}

Stigma; eating disorders; depression; alcohol dependence.

\section{Copyright and usage}

(C) The Author(s), 2021. Published by Cambridge University Press on behalf of the Royal College of Psychiatrists. This is an Open Access article, distributed under the terms of the Creative Commons Attribution licence (https://creativecommons.org/ licenses/by/4.0/), which permits unrestricted re-use, distribution, and reproduction in any medium, provided the original work is properly cited.

\section{Background}

Epidemiological studies suggest that up to $5 \%$ of the population will experience an eating disorder over the course of their lives. ${ }^{1}$ Eating disorders are often accompanied by complex physical and psychiatric comorbidity, and have high mortality rates. ${ }^{1}$ Despite their severity, only a small proportion of individuals with eating disorders seek and obtain help, ${ }^{2}$ medical students still receive minimal training on these conditions ${ }^{3}$ and research funding for eating disorders remains limited. ${ }^{4}$ Despite being a cardinal element of successful public health campaigns, compared with other disorders mental health literacy around eating disorders has received little attention. ${ }^{5}$ Therefore, it is possible that stigma towards eating disorders arising from low levels of mental health literacy around these conditions could account for low levels of help-seeking ${ }^{6}$ as well as the above-mentioned limited research investment and visibility in medical curricula.

Compared with other mental illnesses, studies show that eating disorders are more commonly viewed as less severe, self-inflicted and under an individual's control. ${ }^{7,8}$ Crucially, studies have shown these beliefs are held by professionals ${ }^{9}$ as well as the public. To date, only a limited number of studies have investigated stigmatising attitudes towards people with eating disorders. To the best of our knowledge, all existing studies have used convenience samples, which are likely to be unrepresentative of the general population. In the UK, the Office of National Statistics (ONS) has collected data on stigmatising views held by the public towards seven different mental health conditions in 1998, 2003 and 2008, as part of the Royal College of Psychiatrists' campaign 'Changing Minds: Every family in the land', aimed at reducing stigma towards mental illness. Two previous studies have described findings from the 1998 and 2003 surveys; ${ }^{10,11}$ however, no studies have investigated changes in stigmatising beliefs towards eating disorders over time using the most recent data available (i.e. 2008), or their association with sociodemographic characteristics of respondents controlling for potential confounders in order to identify specific predictors of stigmatising beliefs.

\section{Aims}

Researching the associations of stigmatising beliefs and sociodemographic characteristics may provide evidence of which groups in society to target, given limited resources. The aim of this study was thus to use data from the first and last ONS surveys to investigate whether attitudes towards individuals with eating disorders have changed over time, how these compared with other mental health conditions, and whether stigmatising attitudes are associated with specific sociodemographic characteristics, including gender, ethnicity, age and socioeconomic status. Based on findings from the 2003 survey, we hypothesised that men and those from more deprived socioeconomic positions would endorse more stigmatising beliefs and that the latter would have remained relatively stable over time. ${ }^{10}$

\section{Method}

\section{Participants}

We used data from the July 1998 and July 2008 ONS Omnibus Opinion Surveys. The sampling frame for these surveys were UK 
postal sectors stratified by region and by: proportion of households renting from local authorities, and the proportion in which the head of household was in socioeconomic groups 1-5 or 13. Thirty households were randomly selected in each postal code and, for each household, one adult aged 16 years or over was chosen at random. Participants answered questions on sociodemographic factors by questionnaire, and interviewers asked questions on stigma and mental health aided by the use of flashcards.

\section{Measures of stigma}

The survey asked about participants' views on eight topics that a previous review ${ }^{12}$ had identified as representing core stigmatising beliefs around people with mental illnesses. These were: being dangerous; being unpredictable; being difficult to talk with; having only themselves to blame; being able to pull themselves together; having a poor outcome and responding poorly to treatment. Existing research suggests that these questions have a three-factor structure, consisting of: negative stereotypes, patient blame and perceived inability to recover. ${ }^{13}$

For the purpose of this study, we concentrated on the latter two: patient blame (comprising the statements that people with mental illnesses 'only have themselves to blame' and 'could pull themselves together if they wanted to'), and inability to recover (comprising the statements that people with mental illnesses 'will never recover fully'; and 'will not improve if given treatment'). We chose not to focus on negative stereotypes (i.e. beliefs that people with mental illness are 'dangerous to others' and 'unpredictable') as it was previously shown that a much smaller proportion of respondents endorsed these beliefs with regards to eating disorders compared with other conditions under study. ${ }^{10}$ Each statement was scored on a five-point scale ranging from 'completely disagree' to 'completely agree'.

For all questions, greater scores indicate greater agreement with the statement, for example, placing greater blame on the person or believing that individuals with a given condition have lower ability to improve. We interpreted high scores on questions about blame as representing more stigmatising beliefs around individuals' responsibility for their illness. Questions on ability to recover are thought to tap into respondents' mental health literacy surrounding treatment and also beliefs around severity and chronicity of these conditions. Literature suggests that people with mental health conditions that are perceived as chronic and with poorer prognosis are more likely to be stigmatised and socially rejected. ${ }^{12}$ Therefore, high scores on these questions could identify this type of stigmatising attitudes. However, we also hypothesised that low scores on these questions could index a different type of stigma, for instance, if a certain condition is seen as easier to recover from because it is not considered to be as severe as others. This type of belief might be particularly relevant for conditions that are typically considered as selfinflicted.

In order to understand the nature of, as well as trends in, stigmatising beliefs towards eating disorders, we compared scores of questions relating to individuals with eating disorders with those relating to individuals with depression and alcohol dependence as, based on previous findings, these disorders appeared more comparable with eating disorders than schizophrenia and dementia. ${ }^{10,11}$

\section{Sociodemographic characteristics}

We investigated the following sociodemographic characteristics as potential predictors of stigmatising views: a continuous indicator of participants' age; gender (male/female), education level (compulsory, i.e. age 16 years/ non-compulsory); social class (manual/nonmanual); housing tenure (owning versus renting a house); and ethnicity (White British/ Black, Asian and other minority ethnic groups).

\section{Data analysis}

We described participants' characteristics using means (with standard deviations) and frequencies (with proportions). To describe cross-sectional differences across psychiatric conditions by stigma domain, we used mean scores (with 95\% confidence intervals) across stigma domains by psychiatric condition (i.e. eating disorders, depression, alcohol dependence) and calendar year $(1998,2008)$.

To investigate sociodemographic factors associated with stigma, for each stigma domain we first ran univariable linear regressions, modelling the outcomes (individual stigma domains) as a function of each individual sociodemographic factor. To test for changes in stigma scores between 1998 and 2008, we further included the variable 'year'. We present univariable models in the supplementary material for completeness. In our main analyses, we entered all sociodemographic factors simultaneously in a multivariable model, with stigma domains as the outcome. We did not limit the number of variables in the multivariable model to those with low $P$-values in the univariable model for two reasons. First, if one uses only variables with a $P$-value below a predefined threshold there is a risk of excluding variables that are negatively confounded by other variables subsequently included in the model. Second, we wanted our models to be comparable across conditions. Analyses were based on participants with complete cases and conducted in Stata 15.

\section{Results}

\section{Participants}

A total of 2854 individuals took part in the surveys in 1998 and 2008 and, of these, 2270 (79.5\%) had complete data on all the variables included. We provide a flow chart of study participation in Fig. 1. The majority of the participants were female and of White ethnicity; had only completed compulsory education; had a non-manual occupation; and owned their house (Table 1). The mean age of the sample was 46.9 years (s.d. $=16.9)$. Participants who were older, were from a minority ethnic background, had a non-manual occupation and had only completed compulsory education were more likely to have some missing data (Supplementary Table 1 available at https://doi.org/10.1192/bjp.2021.175). Individuals who took part in the 2008 survey were also more likely to have missing data.

\section{Trends and predictors of stigmatising beliefs around recovery}

In both 1998 and 2008, mean scores for questions relating to whether participants thought that individuals 'would not improve if given treatment' and 'will never recover fully' were lower for eating disorders than severe depression or alcohol dependence (Fig. 2 \& Supplementary Table 2):

(a) eating disorders: 'improvement' question 1998 mean 1.99 (95\% CI 1.94-2.06), 2008 mean 2.10 (95\% CI 2.02-2.18); 'recovery' question 1998 mean 2.26 (95\% CI 2.20-2.31), 2008 mean 2.46 (95\% CI 2.38-2.54);

(b) severe depression: 'improvement' question 1998 mean 2.25 (95\% CI 2.19-2.31), 2008 mean 2.33 (95\% CI 2.24-2.42); 'recovery' question 1998 mean 2.78 (95\% CI 2.72-2.84), 2008 mean 2.92 (95\% CI 2.84-3.00);

(c) alcohol dependence: 'improvement' question 1998 mean 2.16 (95\% CI 2.11-2.23), 2008 mean 2.34 (95\% CI 2.25-2.43); 'recovery' question 1998 mean 2.81 (95\% CI 2.76-2.88), 2008 mean 2.92 (95\% CI 2.82-3.02). 


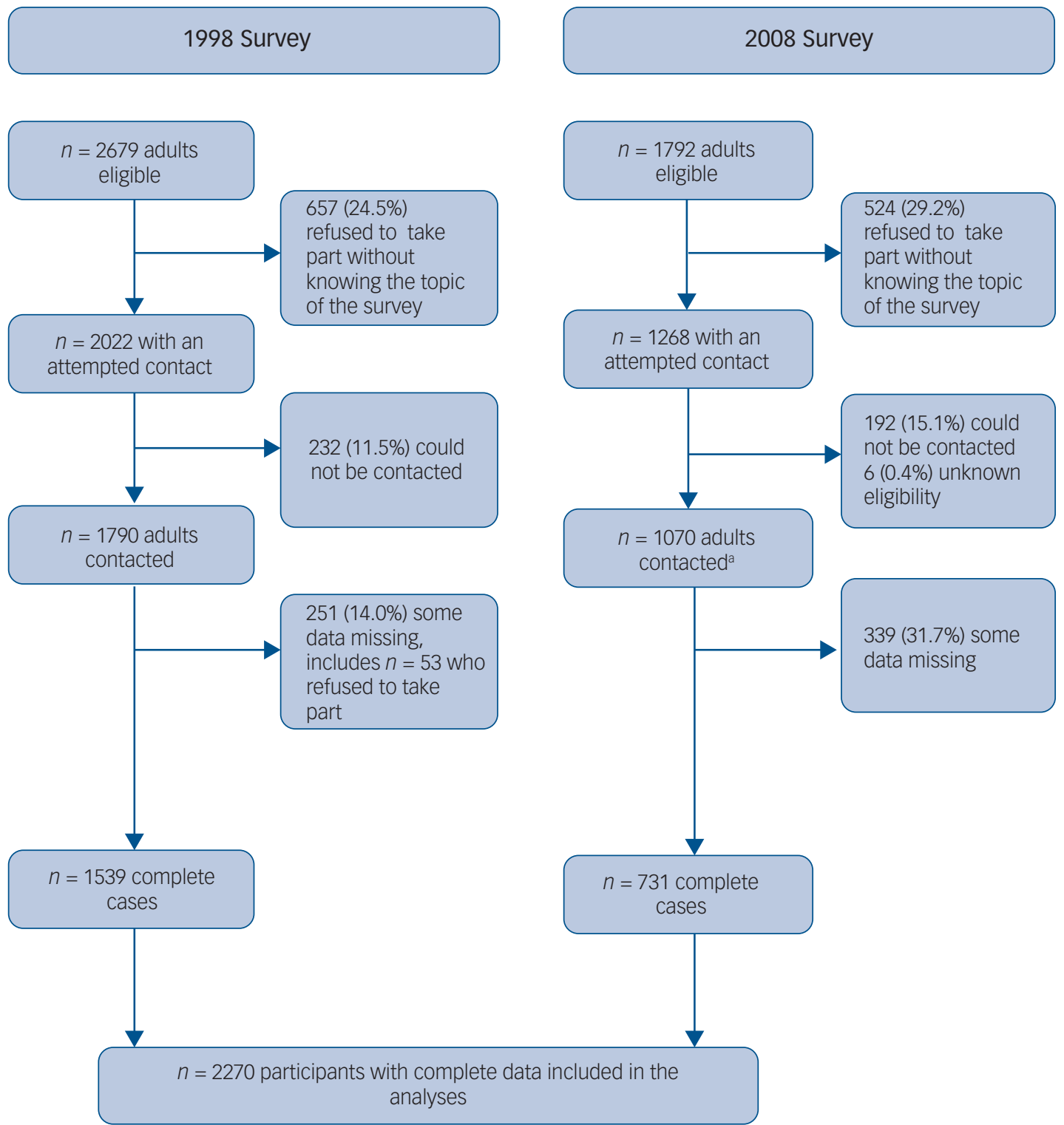

Fig. 1 Flow chart of study participation.

a. Actual data-set included 1064 participants.

\section{Table 1 Sociodemographic characteristics of the participants}

\section{Characteristic}

Total, $n$

Gender, $n$ (\%)

Male

Female

Ethnicity, $n$ (\%)

White

Ethnic minority

Education, $n(\%)$

Compulsory

Non-compulsory

House tenure, $n(\%)$

Own

Rent

Social class, $n$ (\%)

Non-manual

Manual

Year, $n$ (\%)

1998

2008

Age, mean (s.d.)
Value

2270

1007 (44.4)

$1263(55.6)$

2097 (92.4)

$173(7.6)$

1345 (59.3)

925 (40.7)

$1556(68.5)$

$714(31.4)$

$1249(55.0)$

$1021(45.0)$

$1539(67.8)$

$731(32.2)$

$46.9(16.85)$
This suggests that responders believed recovering from eating disorders is easier than recovering from the other conditions. For all three conditions stigmatising beliefs decreased in 2008 compared with 1998 (Fig. 2).

With respect to predictors of these beliefs, we present results of univariable models in Supplementary Table 3 and the main multivariable model in Table 2.

Compared with women, men were more likely to think that people with eating disorders could recover fully (mean difference: $-0.18,95 \%$ CI -0.28 to -0.08 ) and improve with treatment (mean difference: $-0.08,95 \%$ CI -0.18 to 0.01 ) although for the latter association evidence was weaker. We observed a similar pattern for alcohol dependence (not improve with treatment mean difference: $-0.11,95 \%$ CI -0.21 to -0.01 ; not recover fully mean difference: $-0.10,95 \% \mathrm{CI}-0.22$ to -0.01 ), but not depression, for which there was no difference between males and females' beliefs.

Older participants believed that recovery was unlikely across all three mental illnesses. Compared with participants with only compulsory education, those with non-compulsory education 


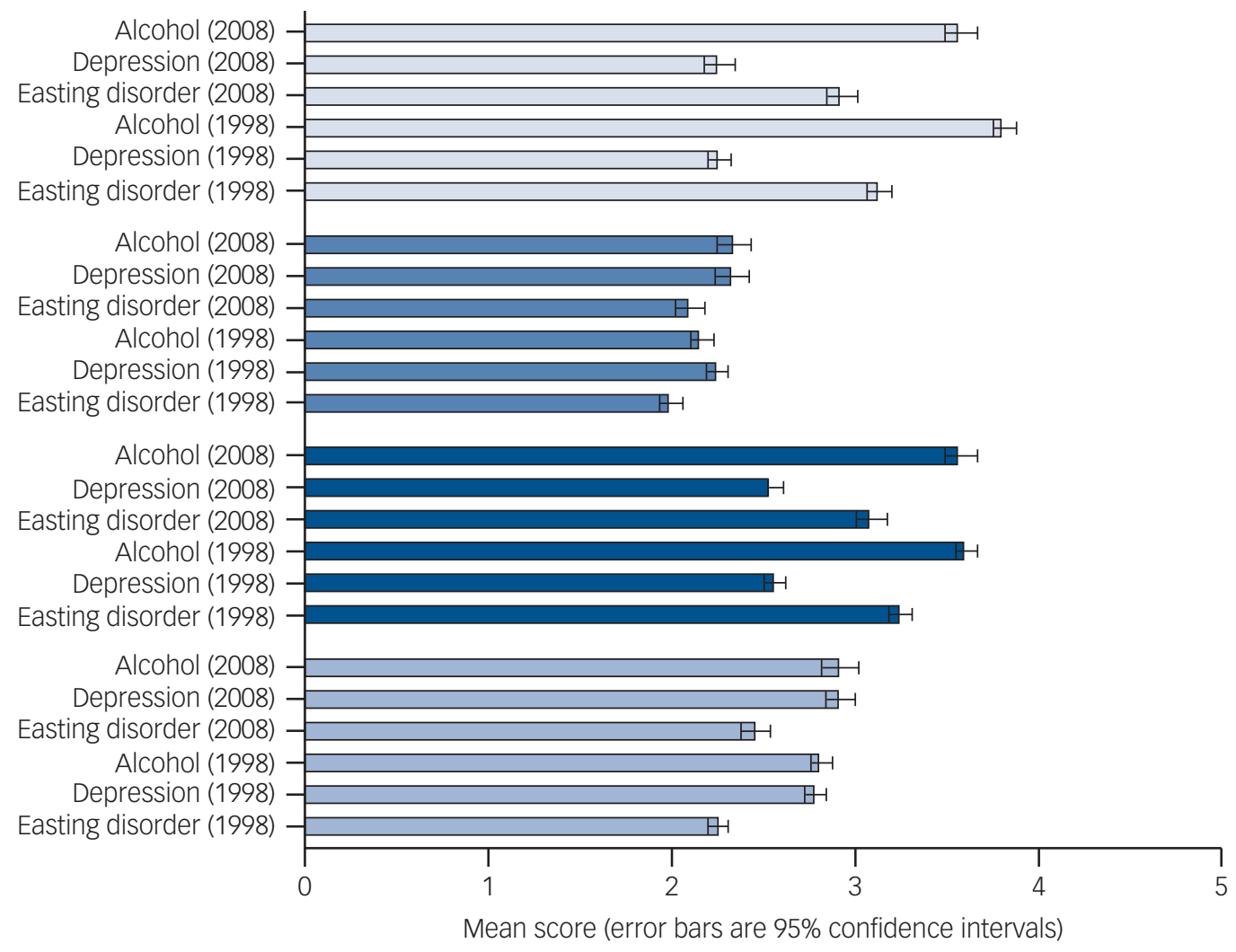
$\square$ People with *condition* have only themselves to blame for it (higher scores indicate more stigmatising belief)
$\square$ People with * condition* would not improve if given treatment (lower scores indicate more stigmatising belief)
People with * ${ }^{*}$ condition* could pull themselves together if they wanted to (higher scores indicate more stigmatising belief)
$\square$ People with * condition* will never recover fully (lower scores indicate more stigmatising belief)

\section{Fig. 2 Mean item score (with 95\% confidence interval) by survey year and psychiatric condition $(n=2270)$.}

more frequently thought that those with depression (mean difference: $-0.23,95 \% \mathrm{CI}-0.35$ to -0.11 ) and alcohol dependence (mean difference: $-0.12,95 \%$ CI -0.24 to -0.01 ), but not those with eating disorders (mean difference: $-0.07,95 \%$ CI -0.17 to 0.04 ) could recover if given treatment. Participants who rented their accommodation more commonly believed that those with eating disorders could not improve if given treatment (mean difference: $0.14,95 \%$ CI $0.03-0.24$ ). The same belief was shared among those with non-manual compared with manual occupations for all three conditions, although evidence was weaker for alcohol dependence. There were no differences in these beliefs by ethnicity.

\section{Trends and predictors of stigmatising beliefs around blame}

In both 1998 and 2008, when participants were asked whether they believed that individuals 'have only themselves to blame for their condition' and 'could pull themselves together if they wanted', they scored eating disorders higher than depression, but lower than alcohol dependence (Fig. 2 and Supplementary Table 2):

(a) eating disorders: 'blame' question 1998 mean 3.13 (95\% CI 3.07-3.20), 2008 mean 2.93 (95\% CI 2.85-3.02); 'pull together' question 1998 mean 3.25 (95\% CI 3.19-3.31), 2008 mean 3.09 (95\% CI 3.01-3.17);

(b) depression: 'blame' question 1998 mean 2.26 (95\% CI 2.202.32), 2008 mean 2.26 (95\% CI 2.18- 2.35), 'pull together' question 1998 mean 2.56 (95\% CI 2.51-2.62), 2008 mean 2.53 (95\% CI 2.45-2.61);

(c) alcohol dependence: 'blame' question 1998 mean 3.81 (95\% CI 3.76-3.88), 2008 mean 3.57 (95\% CI 3.49-3.67), 'pull together' question 1998 mean 3.60 (95\% CI 3.55-3.67), 2008 mean 3.54 (95\% CI 3.46-3.63).

This indicates that respondents believed that people with eating disorders are more to blame for their condition than those with depression, but less than those with alcohol dependence. However, as shown in Fig. 2 (and Supplementary Table 2), compared with 1998, in 2008 stigmatising views towards eating disorders and alcohol dependence improved in these domains. By contrast, for severe depression scores remained similar.

Table 2 shows factors associated with stigmatising views around blame. Compared with women, men expressed more agreement with the idea that people with eating disorders are to blame for their condition (mean difference: $0.24,95 \%$ CI $0.13-$ 0.35), with a comparable association observed for depression 
Table 2 Multivariable linear regression models for the associations between sociodemographic characteristics and stigmatising beliefs, by condition (eating disorders, depression, alcohol dependence) ${ }^{a}$

Mean difference $(95 \% \mathrm{Cl}) P$

Eating disorders Depression Alcohol dependence

People with condition have only themselves to blame for their condition

Gender, male (versus female)

Age

Ethnicity, BAME (versus White)

Year, 2008 (versus 1998)

Education, non-compulsory (versus compulsory)

Social class, non-manual (versus manual)

Housing tenure, rent (versus own)

People with condition will never recover fully

Gender, male (versus female)

Age

Ethnicity, BAME (versus White)

Year, 2008 (versus 1998)

Education, non-compulsory (versus compulsory)

Social class, non-manual (versus manual)

Housing tenure, rent (versus own)

People with condition could pull

Gender, male (versus female)

Age

Ethnicity, BAME (versus White)

Year, 2008 (versus 1998)

Education, non-compulsory (versus compulsory)

Social class, non-manual (versus manual)

Housing tenure, rent (versus own)

People with condition would not improve if given treatment

Gender, male (versus female)

Age

Ethnicity, BAME (versus White)

Year, 2008 (versus 1998)

Education, non-compulsory (versus compulsory)

Social class, non-manual (versus manual)

Housing tenure, rent (versus own)

BAME, Black, Asian, Minority ethnic.

a. Complete case analyses, $n=2270$.
$0.24(0.13$ to 0.35$)<0.0001$

0.09 (0.03 to 0.17$) 0.007$

0.24 (0.01 to 0.48$) 0.047$

$0.25(0.15$ to 0.35$)<0.0001$

-0.04 (-0.10 to 0.02) 0.142

0.62 (0.42 to 0.83 ) $<0.0001$

-0.16 ( -0.28 to -0.04$) 0.010$

$-0.19(-0.32$ to -0.07$) 0.003$

0.06 ( -0.06 to 0.19$) 0.318$

0.04 (-0.10 to 0.17$) 0.590$

$-0.18(-0.28$ to -0.08$)<0.0001$

0.11 (0.05 to 0.17 ) $<0.0001$

-0.02 (-0.24 to 0.20$) 0.845$

0.29 (0.18 to 0.39$)<0.0001$

0.04 (-0.06 to 0.16$) 0.400$

0.08 ( -0.03 to 0.18$) 0.159$

0.08 (-0.03 to 0.19) 0.158

0.37 (0.27 to 0.47$)<0.0001$

-0.07 ( -0.13 to -0.01$) 0.031$

0.37 (0.16 to 0.58 ) 0.001

$-0.20(-0.31$ to -0.10$)<0.0001$

-0.19 ( -0.31 to -0.08$) 0.001$

0.02 (-0.09 to 0.13) 0.722

$0.04(-0.08$ to 0.15$) 0.500$

-0.08 ( -0.18 to 0.01$) 0.098$

0.14 (0.09 to 0.20$)<0.0001$

0.02 ( -0.16 to 0.21$) 0.881$

0.15 (0.05 to 0.25$) 0.004$

-0.07 ( -0.17 to 0.04$) 0.202$

0.13 (0.03 to 0.24$) 0.013$

0.14 (0.03 to 0.24 ) 0.016
-0.01 ( -0.12 to 0.10$) 0.858$

$-0.14(-0.25$ to -0.03$) 0.016$

0.20 (0.09 to 0.31$) 0.001$

-0.09 ( -0.21 to 0.02$) 0.109$

$-0.05(-0.15$ to 0.06$) 0.378$

0.07 (0.01 to 0.13) 0.017

-0.14 (-0.38 to 0.09) 0.229

0.20 (0.09 to 0.30$)<0.0001$

-0.01 ( -0.04 to 0.10$) 0.400$

0.07 ( -0.04 to 0.18$) 0.205$

0.02 ( -0.19 to 0.14$) 0.757$

0.24 (0.14 to 0.34$)<0.0001$

-0.04 (-0.10 to 0.02$) 0.163$

0.42 (0.20 to 0.65$)<0.0001$

-0.01 ( -0.13 to 0.09$) 0.718$

$-0.22(-0.33$ to -0.11$)<0.0001$

$0.10(-0.01$ to 0.21$) 0.062$

0.02 (-0.09 to 0.13$) 0.759$

$-0.03(-0.14$ to 0.08$) 0.599$

$0.12(0.06$ to 0.18$)<0.0001$

0.08 (-0.16 to 0.31$) 0.527$

0.13 (0.01 to 0.25$) 0.030$

$-0.23(-0.35$ to -0.11$)<0.0001$

0.14 (0.02 to 0.26$) 0.020$

$0.11(-0.02$ to 0.24$) 0.110$
0.08 (-0.03 to 0.18) 0.154

-0.01 ( -0.07 to 0.05$) 0.692$

0.07 (-0.05 to 0.29) 0.503

$-0.22(-0.32$ to -0.11$)<0.0001$

$-0.22(-0.33$ to -0.10$)<0.0001$

0.13 (0.02 to 0.25$) 0.020$

0.04 (-0.08 to 0.16) 0.501

$-0.10(-0.22$ to -0.01$) 0.063$

0.16 (0.10 to 0.23$)<0.0001$

-0.20 ( -0.45 to 0.04$) 0.103$

0.14 (0.02 to 0.27$) 0.024$

0.06 ( -0.07 to 0.18$) 0.369$

$-0.08(-0.20$ to 0.05$) 0.224$

0.11 ( -0.02 to 0.24$) 0.088$

0.20 (0.10 to 0.31$)<0.0001$

$-0.12(-0.18$ to -0.06$)<0.0001$

$0.12(-0.09$ to 0.32$) 0.273$

-0.09 ( -0.20 to 0.02$) 0.124$

$-0.18(-0.29$ to -0.07$) 0.001$

0.09 ( -0.02 to 0.20$) 0.121$

0.07 (-0.05 to 0.19$) 0.259$

$-0.11(-0.21$ to -0.01$) 0.037$

$0.18(0.12$ to 0.24$)<0.0001$

0.06 ( -0.18 to 0.30$) 0.610$

$0.27(0.15$ to 0.38$)<0.0001$

$-0.12(-0.24$ to -0.01$) 0.035$

0.10 ( -0.01 to 0.21$) 0.087$

$0.10(-0.02$ to 0.22$) 0.088$ (mean difference: $0.25,95 \%$ CI $0.15-0.35$ ). Men were also more likely than women to think that people with all three mental illnesses could 'pull themselves together' if they wanted to, with the greatest difference observed when referring to people with eating disorders (mean difference: $0.37,95 \%$ CI $0.27-0.47$ ), compared with depression (mean difference: 0.24 , 95\% CI $0.14-0.34$ ) and alcohol dependence (mean difference: 0.20, 95\% CI 0.100.31 ). Older participants believed that people with eating disorders (but not those with depression or alcohol dependence) were to blame for their disorder (mean difference $0.09,95 \%$ CI 0.03-0.17). Older participants also though that people with an eating disorder (mean difference: -0.07 , 95\% CI -0.13 to -0.01 ) and those with alcohol dependence (mean difference: $-0.12,95 \%$ CI -0.18 to -0.06 ) could not 'pull themselves together' they wanted to.

Participants from minority ethnic backgrounds, compared with White participants, were more likely to think that those with eating disorders (mean difference: 0.24 , 95\% CI 0.01-0.48) and depression (mean difference: 0.62 , 95\% CI 0.42-0.83) were to blame for their conditions and that they could 'pull themselves together' if they wanted to (eating disorders mean difference: 0.37 , 95\% CI 0.16-0.58, depression mean difference: 0.42 , 95\% CI 0.200.65). Compared with those with only compulsory levels of education, participants with higher levels of education were less likely to blame individuals for their mental illness (eating disorders mean difference: $-0.19,95 \%$ CI -0.32 to -0.07 ; depression mean difference: $-0.14,95 \% \mathrm{CI}-0.25$ to -0.03 ; alcohol dependence mean difference: $-0.22,95 \%$ CI -0.33 to -0.10 ) or think that they could 'pull themselves together' if they wanted to (eating disorders mean difference: $-0.19,95 \%$ CI -0.31 to -0.08 ; depression mean score: $-0.22,95 \% \mathrm{CI}-0.33$ to -0.11 ; alcohol dependence mean difference: $-0.18,95 \% \mathrm{CI}-0.29$ to -0.07$)$.

\section{Discussion}

\section{Main findings and comparison with the existing literature}

In this study we investigated patterns of stigmatising beliefs towards people with eating disorders, depression and alcohol dependence, how these have changed over 10 years (1998-2008), and how they are associated with sociodemographic factors.

In both 1998 and 2008, respondents thought that people with eating disorders were to be blamed for their conditions more than people with depression, but not more than those with alcohol dependence. Participants also believed that it would be easier to recover from an eating disorder or improve if given treatment, than it would be to recover from either depression or alcohol dependence. These results possibly reflect the widespread belief that eating disorders are under one's control and could be easily recovered from, as previously reported. ${ }^{7,8}$

However, we also found that, over the 10 -year period studied (1998-2008), these stigmatising views towards eating disorders decreased across the board, which was contrary to our hypothesis. This had not been the case when the 1998 results were compared with the results of the 2003 survey (as these only reported 
improvement on the two 'recovery' questions) ${ }^{10}$ and was not observed to the same extent for the other two comparator conditions. This trend is encouraging and is in line with global literature showing improvements in stigma towards mental health diagnoses. Nevertheless, to date this research has primarily focused on depression and schizophrenia and largely overlooked eating disorders, hence comparisons of our findings with those of other countries is difficult. $^{14}$

Finally, we observed that stigmatising views towards people with eating disorders were more common among certain groups, which was broadly in line with our hypothesis. Males, participants who were older or from minority ethnic backgrounds, and those with lower levels of formal education were more likely to attribute blame to people with eating disorders; this pattern was also observed across the other conditions. Older participants were more likely to think that people with eating disorders, as well as other conditions, will never recover fully even if given treatment. On the other hand, compared with women, men were more likely to believe that it was possible to recover from eating disorders and alcohol dependence, but their views did not differ from those of women when considering depression. Previous studies using 1998 and 2003 ONS data found largely similar patterns, although these did not disaggregate between different stigma domains and did not mutually control for sociodemographic factors. ${ }^{10,11}$ Our study therefore offers a more granular understanding of the relationship between different sociodemographic characteristics and specific stigma domains.

\section{Strengths, limitations and direction for future research}

This study has a number of strengths. We used nationally representative general population data spanning a decade, thus avoiding selection biases associated with the use of convenience samples (for example college students). We compared eating disorders with two conditions that are often comorbid with eating disorders and share similar epidemiological profiles (depression) and that we hypothesise would have similar stigma profiles (alcohol dependence), as opposed to disorders that have different stigma and epidemiological profiles, for example dementia or schizophrenia. ${ }^{15}$ We measured our outcomes using continuous scores rather than dichotomising answers, allowing us to investigate our outcomes on a continuum of severity while also achieving good statistical power. Finally, this was the first study to investigate sociodemographic characteristics associated with individual stigma domains and using regression models to control for confounding, thus ensuring robustness of findings.

Despite these strengths, a number of limitations should also be acknowledged. One limitation, as with all large population-level surveys, is that there was some selective attrition that became more prominent in the most recent survey. Although we included predictors of attrition in our analyses, we cannot exclude the possibility that selection bias could have occurred if survey participation was conditional upon certain sociodemographic characteristics, for instance female gender or White ethnicity both of which appear to be over-represented compared with 2011 ONS census figures. ${ }^{16} \mathrm{We}$ did not adjust regression models for multiple comparisons as, given some groups were small (for example ethnic minority participants), attempting to avoid a false positive would have increased the risk of false negatives. Instead, we interpreted the strength of the evidence based on effect size and 95\% confidence intervals, and the majority of the associations observed had very small $P$-values, which suggests there was strong statistical evidence to support a difference.

The survey asked about 'eating disorders' without differentiating between bulimia nervosa, anorexia nervosa or binge eating disorder. As there is some evidence that these conditions may have different stigma profiles, ${ }^{6,8}$ future studies should aim to include questions on individual eating disorders. The survey also asked about 'severe depression' as opposed to 'depression' but did not include any indications of severity for any of the other conditions, which may have biased participants' responses if individuals, or specific sociodemographic groups, perceived this condition as being more severe than the others. The questions around ability to recover did not directly ask about respondents' beliefs around severity of each condition under study and could have measured knowledge around availability of effective treatment options. The lower scores we observe on these questions could have therefore indexed better mental health literacy and fewer stigmatising beliefs. However, we propose that the latter is unlikely as we do observe marked differences between depression and eating disorders, for instance, despite the fact that evidence around effective treatments for eating disorders is weaker than that around effective treatments for depression. Hence, we believe these questions are likely to capture underlying beliefs around severity. Nevertheless, future surveys and studies should include more explicit questions around severity in order to tease apart these two important domains. They should also include questions on participants' weight to explore how the latter might influence people's views on eating disorders.

Although few have addressed eating disorders specifically, in recent years there have been more public health campaigns to increase mental health literacy and decrease stigma, ${ }^{5}$ also helped by public figures talking more openly about their mental health. Although some of the improvement in stigmatising beliefs that we observed could have been because of specific campaigns, our design could not test this, as participants were not asked whether they had been exposed to any specific campaign and, despite covering a 10-year time frame, the data is no longer current. It is therefore important that such surveys continue to be repeated and that evaluation of the effectiveness of anti-stigma and mental health literacy campaigns is embedded in their design in order to understand the impact of anti-stigma and awareness-raising campaigns on trends in mental health stigma. Finally, our results are specific to the UK and might not be generalisable to other countries. It is therefore important that data on mental health stigma is more widely collected so that cross-country comparisons can be conducted.

\section{Implications of our findings}

The finding that males and ethnic minority participants held more stigmatising views towards people with eating disorders has important clinical and research implications. Eating disorders are often framed in the media as White women illnesses. Men who experience these conditions are portrayed as 'atypical' and 'less of a man'. ${ }^{17}$ These negative views could be responsible for the greater levels of stigmatising beliefs we observed in our study and greater internalised stigma seen in males with eating disorders compared with females, which have been reported by previous studies. ${ }^{6}$ However, we found that males had more stigmatising views towards depression and alcohol dependence as well, and this has also been previously observed. ${ }^{18}$ Feelings of shame around experiences of mental illness in males have been shown to hinder help-seeking behaviours ${ }^{19}$ and are thought to be one of the reasons behind their increased risk of suicide. ${ }^{20}$

General population studies suggest that the prevalence of eating disorders and disordered eating in the UK population does not differ across ethnic groups. ${ }^{2}$ However, there is evidence that individuals from Black and Asian minority backgrounds are systematically under-represented in eating disorder services. ${ }^{21}$ This could be explained by lower rates of help-seeking, failure to identify eating disorders in these populations in primary care, or both. Qualitative studies have highlighted that lower mental health literacy and greater stigma towards mental health conditions are barriers towards help-seeking for eating disorders ${ }^{22}$ and mental health problems in general in minority ethnic communities. ${ }^{23}$ 
Interventions aimed at increasing mental health literacy could therefore have multiple benefits - from encouraging greater helpseeking to reducing internalised stigma and improving people's quality of life. For instance, a recent systematic review found that interventions that focused on biological explanations of eating disorders, as opposed to sociocultural ones, were more successful at reducing stigma towards these conditions. ${ }^{24}$ However, the same review also cautioned against excessive reliance on biological explanations as these might give rise to deterministic beliefs around the difficulty of recovering from or preventing an eating disorder. ${ }^{24}$ Our study suggests that, increasing public understanding that eating disorders also affect males and people from ethnic minority backgrounds as part of anti-stigma campaigns could help de-stigmatise them in these groups. It is therefore important that public health campaigns aimed at improving mental health literacy are inclusive and target diverse groups.

In conclusion, we found that although stigmatising views towards eating disorders had improved in 2008 compared with the late 1990s, core differences vis-à-vis other conditions, remained. Although new surveys presenting more current data are needed, our study still provides useful insights on which aspects of eating disorder mental health literacy might need to be addressed by future anti-stigma and mental health literacy campaigns, and which groups might benefit from the latter. Such interventions should be considered a key priority alongside other public health strategies as they are likely to promote greater helpseeking behaviours, improve individuals' well-being and lead to greater investment in research and clinical services, ultimately leading to improved prognosis and opportunities for prevention.

Jennifer Guy, Gloucestershire Health and Care NHS Foundation Trust, UK; Helen Bould (DD. Gloucestershire Health and Care NHS Foundation Trust, UK; Centre for Academic Mental Health, Population Health Science, University of Bristol, UK; and MRC Integrative Epidemiology Unit at the University of Bristol, UK; Glyn Lewis (D), Division of Psychiatry, University College London, UK; Francesca Solmi $\mathbb{D}$, Division of Psychiatry, University College London, UK

Correspondence: Francesca Solmi. Email: francesca.solmi@ucl.ac.uk

First received 27 Jul 2020, final revision 23 Jun 2021, accepted 22 Oct 2021

\section{Supplementary material}

To view supplementary material for this article, please visit https://doi.org/10.1192/bjp.2021.175

\section{Data availability}

The data-sets used in this study are available free of charge on the https://ukdataservice.ac.uk/ website.

\section{Author contributions}

F.S. formulated the research question and designed the study with input from all authors; F.S. analysed the data; J.G. and F.S. drafted the manuscript. All authors contributed to interpreting the results and revising the manuscript.

\section{Funding}

F.S. is funded by the Wellcome Trust (Sir Henry Wellcome Fellowship; grant code: 209196/Z/17/Z). This research is also supported by the UCLH NIHR Biomedical Research Centre.

\section{Declaration of interest}

The authors declare that they have no conflict of interest

\section{References}

1 Treasure J, Claudino AM, Zucker N. Eating disorders. Lancet 2010; 375: 583-93.

2 Solmi F, Hatch SL, Hotopf M, Treasure J, Micali N. Prevalence and correlates of disordered eating in a general population sample: the South East London community health (SELCOH) study. Soc Psychiatry Psychiatr Epidemiol 2014; 49: 1335-46.

3 Ayton A, Ibrahim A. Does UK medical education provide doctors with sufficient skills and knowledge to manage patients with eating disorders safely? Postgrad Med J 2018; 94: 374-80.

4 MQ. UK Mental Health Research Funding 2014-2017. MQ, 2019 (https://www. mqmentalhealth.org/wp-content/uploads/UKMentalHealthResearchFunding 2014-2017digital.pdf).

5 Bullivant B, Rhydderch S, Griffiths S, Mitchison D, Mond JM. Eating disorders "mental health literacy": a scoping review. J Ment Heal 2020; 29(3): 336-49.

6 Griffiths S, Mond JM, Murray SB, Touyz S. The prevalence and adverse associations of stigmatization in people with eating disorders. Int J Eat Disord 2015; 48(6): 767-74.

7 Mond JM, Robertson-Smith G, Vetere A. Stigma and eating disorders: Is there evidence of negative attitudes towards anorexia nervosa among women in the community? J Ment Heal 2006; 15: 519-32.

8 Ebneter DS, Latner JD. Stigmatizing attitudes differ across mental health disorders: a comparison of stigma across eating disorders, obesity, and major depressive disorder. J Nerv Ment Dis 2013; 201(4): 281-5.

9 McNicholas F, O'Connor C, O'Hara L, McNamara N. Stigma and treatment of eating disorders in Ireland: healthcare professionals' knowledge and attitudes. Ir J Psychol Med 2016; 33: 21-31.

10 Crisp A, Gelder M, Goddard E, Meltzer H. Stigmatization of people with mental illnesses: a follow-up study within the changing Minds campaign of the royal college of psychiatrists. World Psychiatry 2005; 4(2): 106-113.

11 Crisp AH, Gelder MG, Rix S, Meltzer HI, Rowlands OJ. Stigmatisation of people with mental illnesses. Br J Psychiatry 2000; Jul(177): 4-7.

12 Hayward P, Bright JA. Stigma and mental illness: a review and critique. J Ment Heal 1997; 6(4): 345-54.

13 Wood L, Birtel M, Alsawy S, Pyle M, Morrison A. Public perceptions of stigma towards people with schizophrenia, depression, and anxiety. Psychiatry Res 2014; 220(1-2): 604-8.

14 Angermeyer MC, Matschinger $\mathrm{H}$, Schomerus $\mathrm{G}$. Attitudes towards psychiatric treatment and people with mental illness: changes over two decades. $\mathrm{Br} J$ Psychiatry 2013; 203(2): 146-51.

15 Hudson Jl, Hiripi E, Pope HG, Kessler RC. The prevalence and correlates of eating disorders in the national comorbidity survey replication. Biol Psychiatry 2007; 61(3): 348-58.

16 Office for National Statistics. 2011 Census Analysis: Ethnicity and Religion of the Non-UK Born Population in England and Wales: 2011. Office for National Statistics, 2015.

17 MacLean A, Sweeting H, Walker L, Patterson C, Räisänen U, Hunt K. 'It's not healthy and it's decidedly not masculine': a media analysis of UK newspaper representations of eating disorders in males. BMJ Open 2015; 5: e007468.

18 Oliffe JL, Ogrodniczuk JS, Gordon SJ, Creighton G, Kelly MT, Black N, et al. Stigma in Male Depression and Suicide: A Canadian Sex Comparison Study. Community Ment Health J 2016; 52(3): 302-310.

19 Griffiths S, Mond JM, Li Z, Gunatilake S, Murray SB, Sheffield J, et al. Self-stigma of seeking treatment and being male predict an increased likelihood of having an undiagnosed eating disorder. Int J Eat Disord 2015; 48(6): 775-8.

20 Rasmussen ML, Hjelmeland $\mathrm{H}$, Dieserud G. Barriers toward help-seeking among young men prior to suicide. Death Stud 2018; 42(2): 96-103.

21 Abbas S, Damani S, Malik I, Button E, Aldridge S, Palmer RL. A comparative study of South Asian and non-Asian referrals to an eating disorders service in leicester, UK. Eur Eat Disord Rev 2010; 18: 404-9.

22 Wales J, Brewin N, Raghavan R, Arcelus J. Exploring barriers to South Asian help-seeking for eating disorders. Ment Heal Rev J 2017; 22: 40-50.

23 Memon A, Taylor K, Mohebati LM, Sundin J, Cooper M, Scanlon T, et al. Perceived barriers to accessing mental health services among black and minority ethnic (BME) communities: a qualitative study in Southeast England. BMJ Open 2016; 6: e012337.

24 Doley JR, Hart LM, Stukas AA, Petrovic K, Bouguettaya A, Paxton SJ. Interventions to reduce the stigma of eating disorders: a systematic review and meta-analysis. Int J Eat Disord 2017; 50: 210-30.

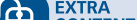

